\title{
IncRNA Xist Regulates Osteoblast Differentiation by Sponging miR-19a-3p in Aging-induced Osteoporosis
}

\author{
Shijie Chen ${ }^{1,2}$, Yuezhan Li ${ }^{1}$, Shuang Zhi ${ }^{4}$, Zhiyu Ding ${ }^{1}$, Yan Huang ${ }^{5}$, Weiguo Wang ${ }^{1}$, Ruping \\ Zheng ${ }^{6}$, Haiyang Yu${ }^{6}$, Jianlong Wang', Minghua $\mathrm{Hu}^{3}$, Jinglei Miao ${ }^{*}$, Jinsong $\mathbf{L i}^{1^{*}}$ \\ ${ }^{1}$ Department of Orthopaedics, The Third Xiangya Hospital of Central South University, Changsha, China. \\ ${ }^{2}$ Shanghai Key Laboratory of Regulatory Biology, Institute of Biomedical Sciences and School of Life Sciences, \\ East China Normal University, Shanghai, China. ${ }^{3}$ Department of Anatomy, Histology and Embryology, Changsha \\ Medical University, Changsha, China. ${ }^{4}$ Four Gynecological Wards, Ningbo Women \& Children's Hospital, \\ Ningbo, Zhejiang, China. ${ }^{5}$ The Second Xiangya Hospital of Central South University, Changsha, China. ${ }^{6}$ School \\ of Basic Medical Science, Central South University, Changsha, China.
}

[Received April 18, 2019; Revised July 10, 2019; Accepted July 24, 2019]

\begin{abstract}
The switch between osteogenic and adipogenic differentiation of bone marrow mesenchymal stem cells (BMSCs) plays a key role in aging-induced osteoporosis. In this study, miR-19a-3p was obviously downregulated in BMSCs from aged humans and mice. Overexpressed miR-19a-3p evidently reduced aginginduced bone loss in mice and promoted osteogenic differentiation of BMSCs, while silenced miR-19a-3p manifestly increased aging-induced bone loss in mice and repressed osteogenic differentiation of BMSCs. Hoxa5 was significantly downregulated in the BMSCs from aged mice and contribute to miR-19a-3p-induced osteoblast differentiation as a direct target gene of miR-19a-3p. Furthermore, IncRNA Xist was found as a sponge of miR19a-3p to repress BMSCs osteogenic differentiation. In conclusion, our study reveals the critical role of the IncRNA Xist/miR-19a-3p/Hoxa5 pathway in aging-induced osteogenic differentiation of BMSCs, indicating the potential therapeutic target for osteoporosis.
\end{abstract}

Key words: Osteoporosis, BMSCs, miR-19a-3p, Hoxa5, lncRNA Xist

Osteoporosis is a frequent age-related disease with severe bone loss, that affects millions of people in the world [1]. BMSCs could be differentiate into osteoblasts and adipocytes, and the switch showed more tendency to osteoblast with age, resulting in age-related marrow fat accumulation and progressive bone loss [2, 3]. It is, therefore, urgent for revealing the underline molecular mechanisms in aging-induced BMSCs differentiation.
MicroRNAs (miRNAs, with 18-24 nucleotides), a class of small noncoding RNAs that are reported to regulate multiple biological processes, including BMSCs differentiation. For example, Guo et al. revealed that the miR-23a/b was downregulated in aged BMSCs and contribute to osteogenic differentiation of BMSCs [4]. miR-204 was reported to be an attenuator of Runx2 and promote adipocyte differentiation of BMSCs

*Correspondence should be addressed to: Drs. Jinglei Mia and Jinsong Li, Department of Orthopaedics, The Third Xiangya Hospital of Central South University, Changsha, China. Email: jinsongli_csu@163.com (JL). miaojinglei@126.com (JM).

Copyright: ( 2019 Chen S et al. This is an open-access article distributed under the terms of the Creative Commons Attribution License, which permits unrestricted use, distribution, and reproduction in any medium, provided the original author and source are credited. 
[5]. miR-188 is evidently upregulated in aged BMSCs and induced age-related BMSCs adipocyte differentiation [3]. Despite these findings, the involvement of miRNA in BMSCs differentiation during aging-induced osteoporosis is still limited. miR-19a-3p (miR-19a) was reported as to be broadly conserved among vertebrates [6]. It was reported to participate in the pathogenesis of preeclampsia and atherosclerosis [7, 8]. A recent study has demonstrated the involvement of miR-19a-3p in the progression of various cancers including glioma, lung cancer, breast cancer, osteosarcoma, gastric cancer and hepatocellular carcinoma [9-13]. However, the precise mechanism by which miR-19a-3p in aging-induced osteoporosis remains unknown.

In this study, we identified that miR-19a-3p downregulated in the BMSCs of aged mice and humans and involves osteoporosis via promoting osteoblast differentiation from BMSCs by targeting Hoxa5. We also demonstrated that lncRNA Xist as the sponge of miR-19a$3 p$ involved in osteoblast differentiation. In conclusion, our study clarified the Xist/miR-19a-3p/Hoxa5 in agerelated BMSCs differentiation, and thus, might provide potential therapeutic approach for osteoporosis.

\section{MATERIALS AND METHODS}

\section{Animals and Clinical samples}

For miR-19a-3p ${ }^{-1}$ mice, we used CRISPR/Cas9 technology. In brief, we used the primer sequences of the
miR-19a-3p F, 5'-CCCTGCTCCCTCTCTCAC-3', and R, 5'-CAGAGAGCTCACCCTC-3' for sgRNA plasmid. The mRNA was obtained from plasmid using the mMESSAGE (Life Technologies). The synthesized mRNAs were purified and injected to the zygotes from C57BL/6 mice as previous described [3]. For miR-19a$3 p \mathrm{Tg}$ mice, we synthesized pre-mir-19a-3p cDNA and cloned to an osterix vector to build an osterix-pre-miR$19 \mathrm{a}-3 \mathrm{p}$ plasmid. The plasmid was transfected into BMSCs and build the osteoprogenitor-specific miR-19a-3p transgenic mouse as previous described [3].

The bone marrow tissues were obtained from 61 female and 62 male patients with osteoarthritis undergoing knee joint replacement or with femoral neck fracture and/or femoral head fractures undergoing hip joint replacement, (with age from 20 to 85) from the Department of Orthopaedics of the Third Xiangya Hospital. The present study was approved by the e Animal Care and Use Committee and Ethics Committee of the Third Xiangya Hospital of Central South University.

\section{BMSC Culture and Transfection}

The BMSCs were isolated from mouse and human as previous described [4, 14]. The agomiR-19a-3p and antagomiR-19a-3p, the plasmid and siRNA of Hoxa5 and Xist were synthesized from Genepharma (Suzhou, China). agomiRNA, antagomiRNA, plamis and siRNA were transfected to BMSCs as previous described [4].

Table 1. The primers.

\begin{tabular}{|c|c|c|}
\hline & Forward $5^{\prime}-3^{\prime}$ & Reverse $5^{\prime}-3^{\prime}$ \\
\hline$\beta$-actin & CTGTCCCTGTATGCCTCTG & TGATGTCACGCACGATTT \\
\hline Runx2 & ACTTCCTGTGCTCCGTGCTG & TCGTTGAACCTGGCTACTTGG \\
\hline Hoxa5 & CTCATTTTGCGGTCGCTATCC & ATCCATGCCATTGTAGCCGTA \\
\hline Osterix & ACCAGGTCCAGGCAACAC & GCAAAGTCAGATGGGTAAGTAG \\
\hline miR-19a-3p & CTGGAGTGTGCAAATCTATGC & GTGCAGGGTCCGAGGT \\
\hline LncRNA Xist & AATGGAACGGGCTGAGTTTTAG & TCATCCGCTTGCGTTCATAG \\
\hline U6 & GCGCGTCGTGAAGCGTTC & GTGCAGGGTCCGAGGT \\
\hline Antagomir-19a-3p & TCAGTTTTGCATAGATTTGCACA & \\
\hline antagomir NC & CAGUACUUUUGUGUAGUACAA & \\
\hline agomir-19a-3p & UGUGCAAAUCUAUGCAAAACUGA & \\
\hline pGL3-HOXA5 & GGGGTACC GCATCTGAGCG & CCGCTCGAG GCTGATCACAGTT \\
\hline pGL3-Xist & GGGGTACCCGGCTTGCTCCA & CCGCTCGAG TTCAAAACAAAGCA \\
\hline pcDNA3.1-HOXA5 & GGGGTACC ATGAGCTCTTATT & CCGCTCGAG TCAGGGGCGGAA \\
\hline pcDNA3.1-Xist & GGGGTACCCGGCTTGCTCCA & CCGCTCGAG TTCAAAACAAAGCA \\
\hline SiHOXA5 & GCACATTAGTCACGACAAT & \\
\hline si_control & GCATTAGTCACGACCAAAT & \\
\hline siXist & GCACACATCTCATTCCATT & \\
\hline
\end{tabular}




\section{Microcomputed Tomography Analysis.}

The right femora from young and old mice were scanned and analyzed by high-resolution mCT (Skyscan 1172, Skyscan). For the distal femur, we selected the region of interest (ROI) for detecting the trabecular number (Tb. N), trabecular thickness ( $\mathrm{Tb}$. Th), trabecular separation (Tb. $\mathrm{Sp}$ ), trabecular bone volume per tissue volume ( $\mathrm{Tb}$. $\mathrm{BV} / \mathrm{TV})$. To examine dynamic bone formation, calcein double labelling was used for trabecular bone, bone formation rates (BFRs) and mineral apposition rate (MAR) as previous described [14].

\section{Osteogenic Differentiation}

$5 \times 10^{5}$ BMSCs were seeded into 24-well plates to induce osteogenic differentiation using osteogenic-inducing medium as previous described. We used the alkaline phosphatase (ALP) kit (Roche Diagnostics, Minneapolis, MN, USA) for ALP activity assay and used the specific immunoassay kit (DiaSorin, Stillwater, MN, USA) for detecting osteocalcin levels. Alizarin Red staining was used to detected osteoblastic mineralization of BMSCs as previous described [14].

\section{qRT-PCR Analysis}

qRT-PCR was used for detecting gene, miRNA or lncRNA expression as previously described [15]. The nucleotide sequences of primers for miR-19a-3p, and U6, Runx2, Osterix, ITGB3 and $\beta$-actin are listed in Table 1.

\section{Western Blot}

Total protein was obtained from BMSCs and then separated using SDS-PAGE, and then incubated anti-
Hoxa5 antibody (sigma, USA) on PVDF membranes (Millipore) $4{ }^{\circ} \mathrm{C}$ overnight followed by HRP conjugated secondary antibody for 1 hour at room temperature. The protein expression levels were analyzed using using ChemiDoc XRS + Imaging System (BioRad).

\section{Dual Luciferase Reporter Assay}

We cloned the 3'- UTR of Hoxa5 into pGL3 luciferase reporter vector (Promega, Madison, WI, USA). And the mut-HOXA5 pGL3 was obtained by site-directed mutagenesis method. The HOXA5-pGL3 or mutHOXA5pGL3 was contransfected with agomiR-19a-3p or agomiR-NC, or antagomiR-19a-3p or antagomiR-NC into BMSCs and the Dual-Luciferase Reporter Assay System (Promega) was used to detecte the luciferase activities.

For the relationship between Xist and miR-19a-3p, we cloned the potential binding site between miR-19a-3p and Xist to pGL3 vector, and then build a mutant XISTpGL3 using site-directed mutagenesis method. The XISTpGL3 or mutXIST-pGL3 was contransfected with agomiR-19a-3p or agomiR-NC, or antagomiR-19a-3p or antagomiR-NC into BMSCs and the luciferase activities was detected using Dual-Luciferase Reporter Assay System (Promega).

\section{RNA Immunoprecipitation (RIP) Assay}

pMS2bp-GFP and MS2, MS2-XIST, or MS2- mutXIST were contransfected to BMSCs cells, and harvested at 48 hours after transfection. The biotin-coupled RNA complex was pulled down and was used for RIP assay using Magna RIPTM Kit (Millipore, USA), according to the manufacturer's instructions [16]. The anti-GFP and anti-IgG were used to incubate, and qPCR was used for the miR-19a-3p level.
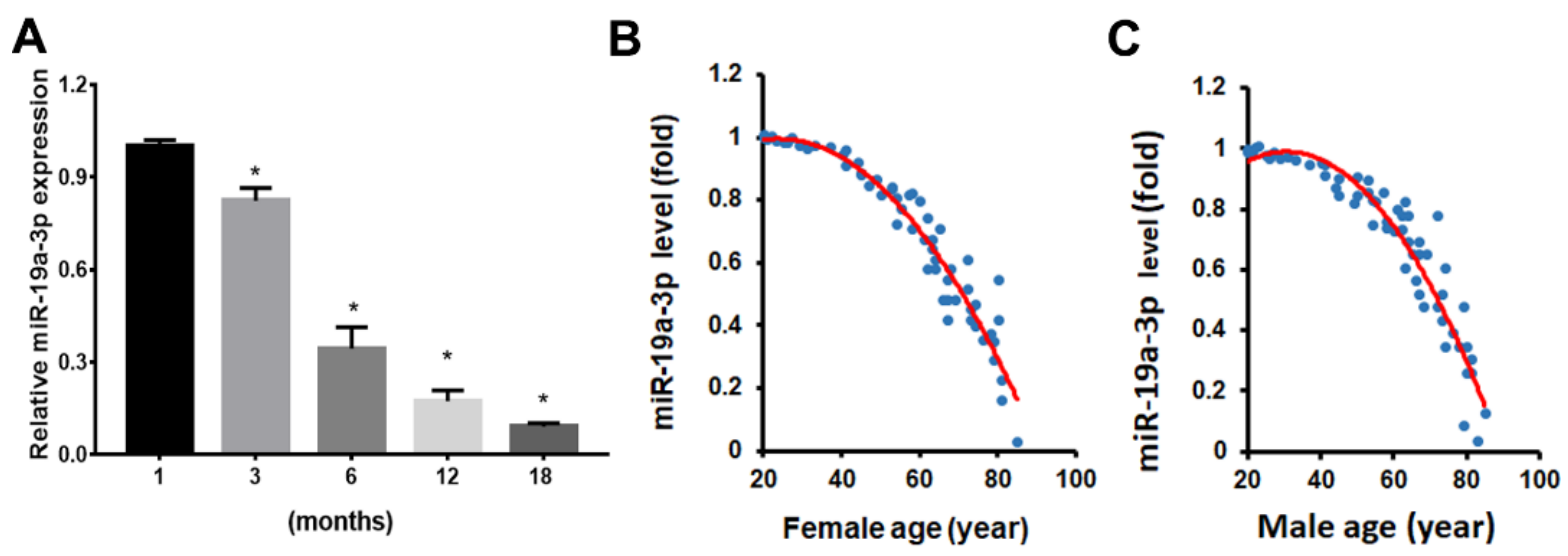

Figure 1. Aging induces miR-19a-3p expression in BMSCs. A The miR-19a-3p expression in BMSCs from mice with different ages. B, The relationship between miR-19a-3p expression with age in BMSCs from 61 human females and C, 62 males. Data shown as mean \pm SD. $* p<0.05$. 


\section{RNA Pull-down Assay}

The capture of miR-19a-3p- bound lncRNAs in a pulldown assays with biotinylated miR-19a-3p and biotinylated lncRNA Xist were performed as previously described[16]. The Biotin-labeled miR-19a-3p and Xist were transfected into BMSCs. After $72 \mathrm{~h}$, BMSCs were harvested and the biotin-coupled RNA complex was pulled down by incubating the cell lysates with M-280 streptavidin magnetic beads (Sigma) for binding at $4{ }^{\circ} \mathrm{C}$ for at least $3 \mathrm{~h}$. And then, the beads bounded with RNA was purified by TRIzol. qPCR assay was used for detecting Xist or miR-19a-3p levels.

\section{FISH}

Briefly, FITC-labeled Xist and Cy3-labeled miR-19a-3p were designed and synthesized by Songan Biotech. The probe signals were detected using a situ hybridization kit (RiboBio) for FISH assay according to the manufacturer's instructions as previous described [17]. The Leica SP5 confocal microscope (Leica Microsystems) was used for confocal images.

\section{Statistical Analyses}

All data were analyzed using SPSS 22.0 and presented as the mean \pm s.d. A Student's $t$-test was used for the comparisons of two groups. One-way ANOVA was used for the comparisons of multiple groups. $P<0.05$ presents statistically significant.

\section{RESULTS}

\section{miR-19a-3p Expression in BMSCs with Age}

Previous studies showed that BMSCs observed more tendency to adipocytes differentiate with age. To investigate the dysregulated miRNA expression in aged BMSCs, we analyzed the miRNA expression from GEO database (accession number GSE57127) and found that miR-19a-3p were downregulated in aged BMSCs. We next verified the miR-19a-3p expression in BMSCs from the mice with different ages using qPCR assay (Fig. 1A). We confirmed the decreased miR-19a-3p levels in human BMSCs with aging and revealed the negative relationship between miR-19a-3p expression and age (Fig. 1B and C). This result revealed the potential important role of miR$19 \mathrm{a}-3 \mathrm{p}$ in the aging process BMSCs.
A

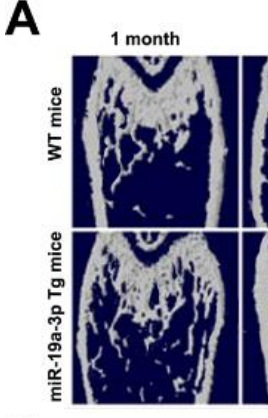

D

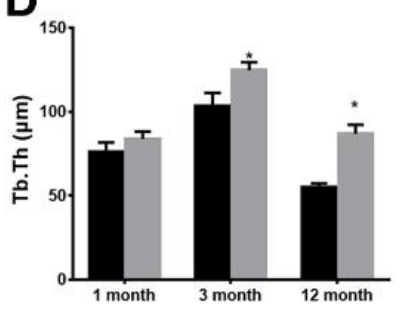

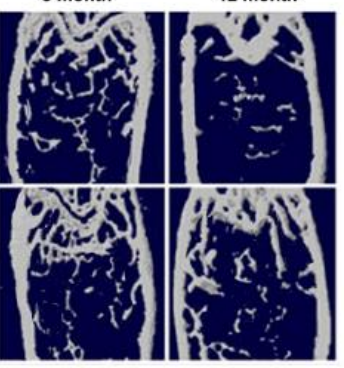

E

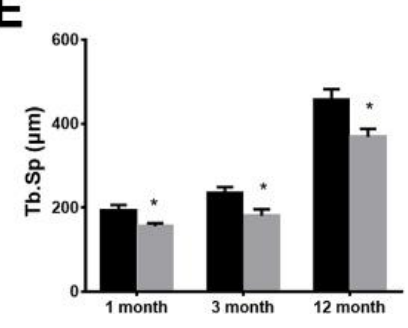

B

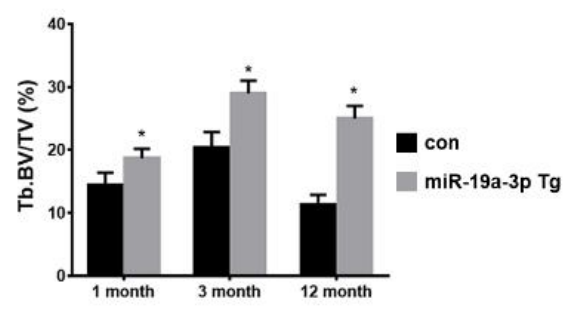

$\mathbf{F}$

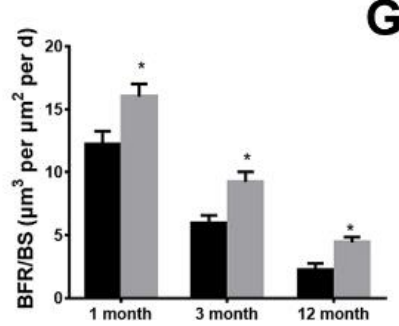

C

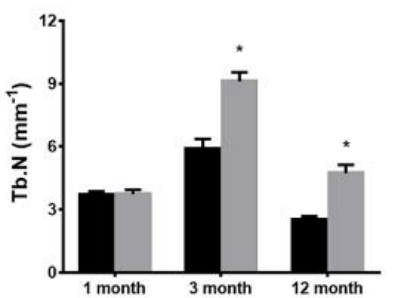

G

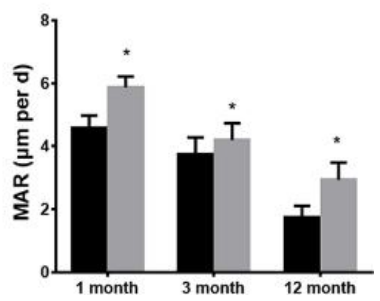

Figure 2. The age-associated bone loss in miR-19a-3p Tg mice. (A) The $\mu \mathrm{CT}$ images of WT mice and miR-19a-3p Tg mice at 1,3 and 12 months old. B-E, quantitative $\mu \mathrm{CT}$ analysis of trabecular bone microarchitecture. (B) Tb. BV/TV, trabecular bone volume per tissue volume. (C) Tb.N, trabecular number. (D) Tb.Th, trabecular thickness. E, Tb.Sp, trabecular separation. (F, G) Representative images of calcein double labelling of trabecular bone, $(\mathbf{G})$ with quantification of BFR per bone surface BFR/BS, H, and mineral apposition rate MAR. Data shown as mean $\pm \mathrm{SD}, * \mathrm{p}<0.05$. 


\section{Overexpressed miR-19a-3p attenuates age-associated bone loss}

To explore the involvement of miR-19a-3p in age-related osteoporosis, the 1, 3, 12 months old WT mice and transgenic overexpressing miR-19a-3p (miR-19a-3p Tg) mice was used to detected aged-associated bone loss using Microcomputed tomography $(\mu-\mathrm{CT})$. We found that the trabecular bone volume, number and thickness were significantly increased, and the trabecular separation was evidently decreased in miR-19a-3p Tg mice of aged (3 or12 months) compared to WT controls (Fig. 2A-E). Moreover, miR-19a-3p Tg mice showed increased bone formation rates (BFRs; Fig. $2 \mathrm{~F}$ and $\mathrm{G}$ ). These results suggested that highly expressed miR-19a-3p attenuates the aging-induced bone loss.

\section{Knockout miR-19a-3p Induces Age-associated Bone Loss}

We next used knockout mice (miR-19a-3p ${ }^{-1-}$ mice) to investigate whether silenced miR-19a-3p induces ageassociated bone loss in vivo. As shown in Figure 3A-E, the trabecular bone volume, the trabecular bone number and the trabecular bone thickness were significantly decreased in the femora of 3-month-old and 12-month-old miR-19a-3p mice relative to WT controls. The trabecular separation was evidently increased in miR-19a$3 \mathrm{p}^{-/}$mice of aged (3 or 12 months) compared to WT controls. Moreover, bone formation rates were also reduced in miR-19a-3p ${ }^{-1}$ mice as compared with WT controls (Fig. 3F and $\mathrm{G}$ ). Together, our results suggested that silenced miR-19a-3p promoted the aging-induced bone 1 .
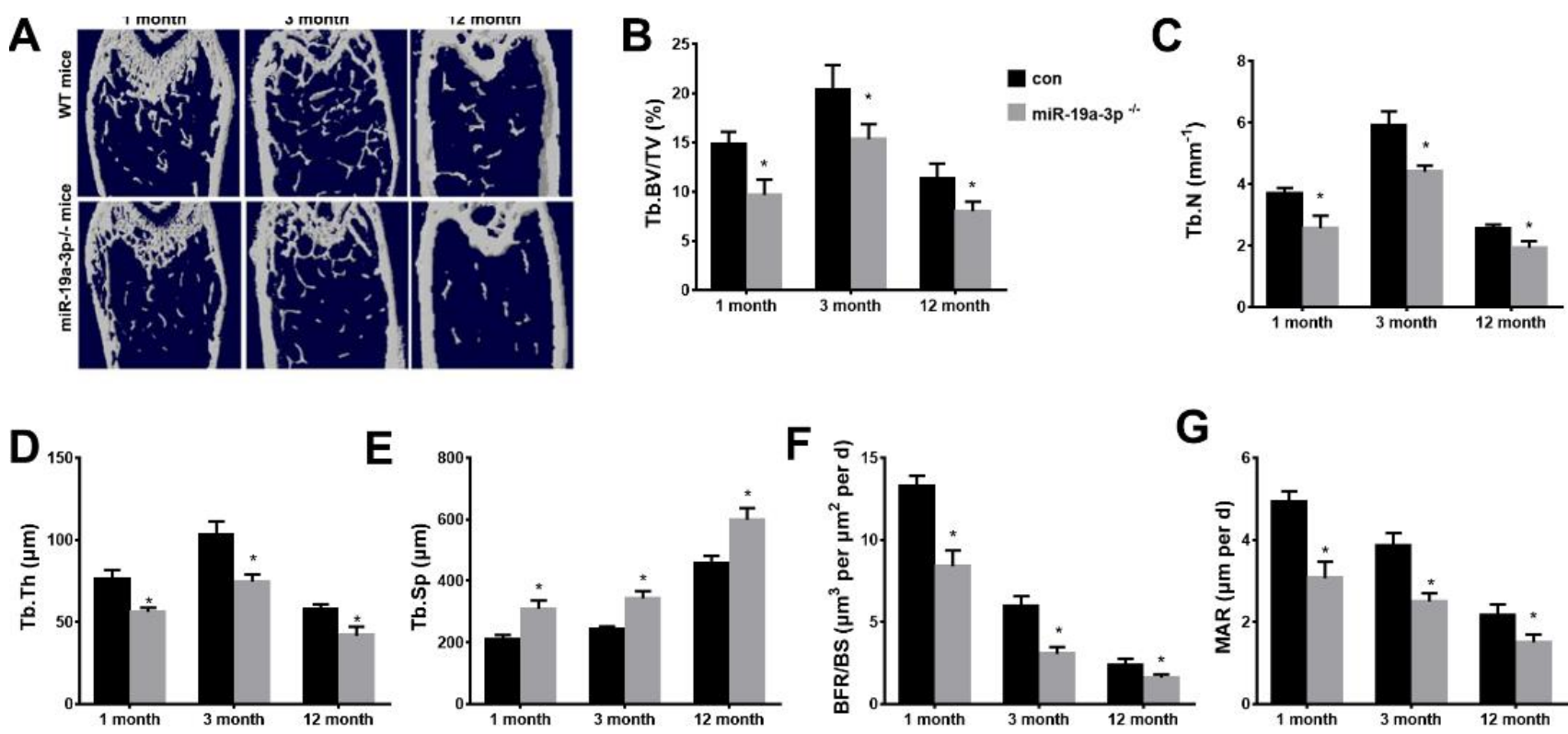

Figure 3. Age-associated bone loss in miR-19a-3p knockout mice. (A) $\mu \mathrm{CT}$ images of WT mice and miR-19a-3p ${ }^{-/-}$mice at 1,3 and 12 months old. B-E, quantitative the trabecular bone microarchitecture. (B) Tb. BV/TV, trabecular bone volume per tissue volume. (C) Tb.N, trabecular number. (D) Tb.Th, trabecular thickness. (E) Tb.Sp, trabecular separation. (F, G) quantification of BFR/BS and MAR. (F) with quantification of BFR per bone surface (BFR/BS); (G) mineral apposition rate (MAR). Data shown as mean \pm SD. *p $<0.05$.

\section{miR-19a-3p Regulates the BMSCs Osteogenic Differentiation}

We next detected role of miR-19a-3p on osteogenic differentiation of BMSCs. As shown in Figure 4A, miR$19 a-3 p$ expression was gradually increased during osteogenic differentiation in the BMSCs from 8 weeksold mice. We next silenced or overexpressed miR-19a-3p in BMSCs using antagomiR-19a-3p or agomiR-19a-3p (Fig. 4B). Alizarin Red staining demonstrated the reduction of osteogenic differentiation in miR-19a-3p silenced BMSCs, whereas the increase of osteogenic differentiation in the miR-19a-3p overexpressed BMSCs (Fig. 4C and D). Consistently, the markers of osteoblast differentiation, including ALP activity, osteocalcin secretion, osteoblast transcription factor osterix were increased by overexpressed-miR-19a-3p but decreased by silenced-miR-19a-3p (Fig. 4E-H). However, overexpressed-miR-19a-3p or silenced-miR-19a-3p did not significant affect the expression of RUNX2 in BMSCs (Fig. 4G). These results indicated that miR-19a-3p promotes the osteogenic differentiation in BMSCs. 


\section{miR-19a-3p Directly Targets Hoxa5}

miRNAs regulate mRNAs expression by binding to the 3 'UTRs of mRNA [13]. We next used microrna (www.microrna.org/microrna/home.do) and targetscan (/www.targetscan.org) to predict the target genes of miR19a-3p. We found that homeobox protein A5 (Hoxa5), a regulator of osteogenic differentiation, was predicted target of miR-19a-3p (Fig. 5A), so we choose Hoxa5 for the further analysis. Next, we used a luciferase reporter assay to clarify the role of miR-19a-3p on Hoxa5. As shown in Fig. 5B, agomiR-19a-3p evidently reduced the luciferase activity of WT-pGL3-HOXA5 but fail to affect the luciferase activity of MUT-pGL3-HOXA5 in BMSCs. antagomiR-19a-3p increased the luciferase activity of WT-pGL3-HOXA5 but failed to affect the luciferase activity of MUT-pGL3-HOXA5 in BMSCs. These results confirmed that miR-19a-3p targets Hoxa5 at its 3 '-UTR directly. We further determined the role of miR-19a-3p on Hoxa5 expression. We found that silenced miR-19a-3p evidently elevated both mRNA and protein expression levels of Hoxa5, whereas overexpressed miR-19a-3p repressed the mRNA and protein levels of Hoxa5 (Fig. 5C). Moreover, Hoxa5 expression was increased in BMSCs of aged mice (Fig. 5D). The Hoxa5 expression was downregulated in the BMSCs from aged (12 months) miR-19a-3p tg mice and upregulated in the BMSCs from aged (12 months) KO mice (Supplementary Fig. 1A).

We next determine the role of Hoxa5 on miR-19a-3pmediated repression of osteogenic differentiation in BMSCs. We found that overexpressed Hoxa5 reduced ALP activity and osteocalcin secretion (Fig. 5E and G), overexpressed Hoxa5 reduced agomiR-19a-3p-induced ALP activity and osteocalcin secretion in BMSCs. Moreover, silenced Hoxa5 markedly induced ALP activity and silenced Hoxa5 reversed antagomiR-19a-3pmediated repression of ALP activity and osteocalcin secretion (Fig. 5F and $\mathrm{H}$ ). In conclusion, these data indicated that miR-19a-3p regulates age-associated BMSC differentiation by targeting Hoxa5 expression directly.

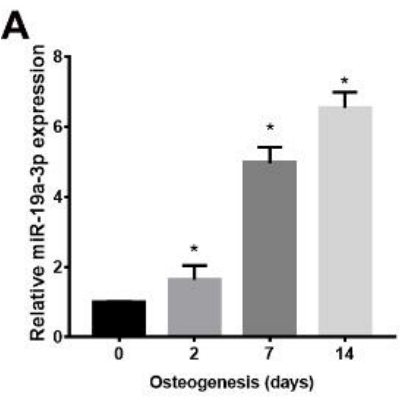

E

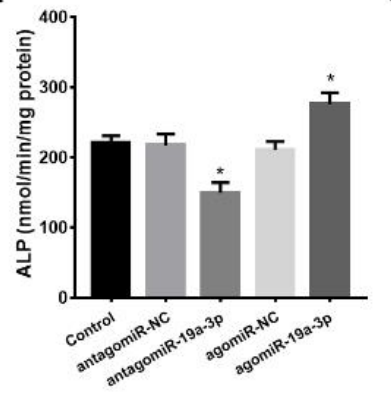

B

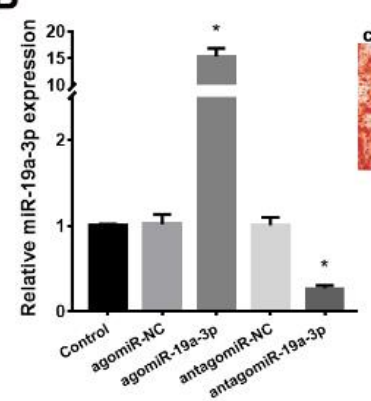

$F$

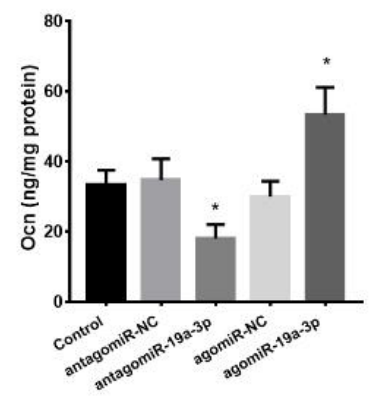

C
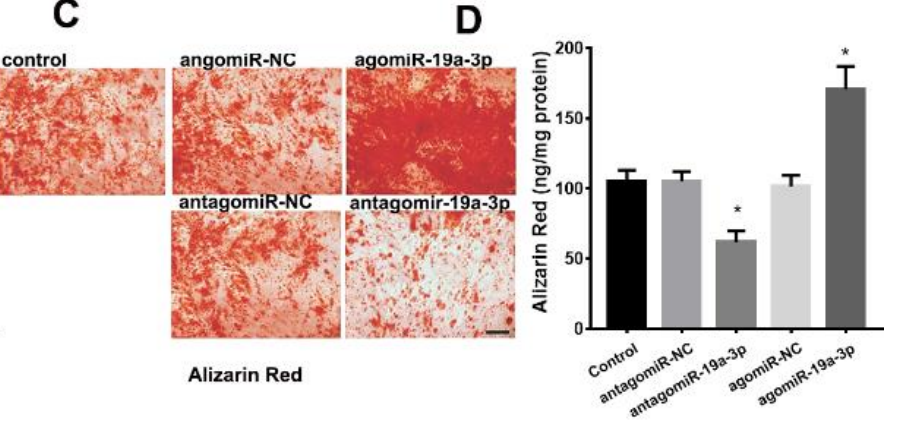

G

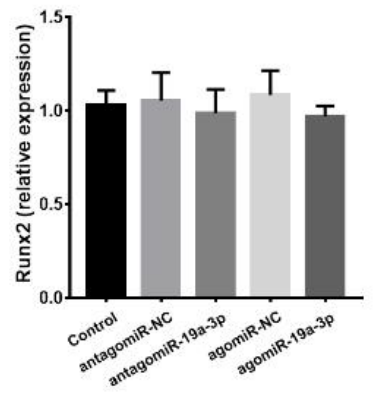

H

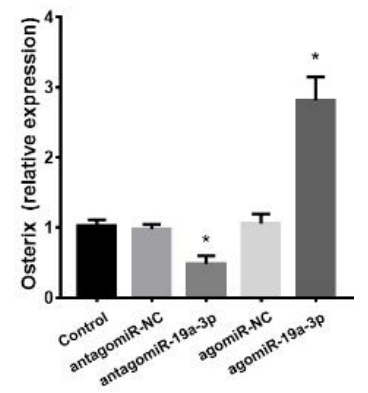

Figure 4. miR-19a-3p regulates the osteogenic differentiation in BMSCs. (A) miR-19a-3p expression in the BMSCs during osteogenic differentiation. (B) The antagomiR-19a-3p or agomiR-19a-3p were used to silence or overexpress miR-19a-3p in BMSCs. (C, D) Alizarin Red staining revealed the role of miR-19a-3p on the osteogenic differentiation of BMSCs. Scale bars: $100 \mu \mathrm{m}$. (E) ALP activity, (F) osteocalcin secretion, (G) RUNX2 and H, osterix expression in BMSCs. Data shown as mean \pm SD. $* p<0.05$.

\section{IncRNA Xist Acted as a Sponge of miR-19a-3p}

We next predict the IncRNA which may bind to miR-19a$3 p$ using starBase. As shown in Figure 6A, miR-19a-3p may be targeted by lncRNA Xist. The lncRNA Xist expression in BMSCs with different age was detected using qPCR analysis. As the animals aged, lncRNA Xist expression was increased in BMSCs (Fig. 6A). RNA FISH revealed the colocalization of IncRNA Xist and miR-19a-3p in the cytoplasm of BMSCs in Figure 6B. We 
performed RNA pull-down assay and RNA immuneprecipitation (RIP) assay to further verify the relationship between miR-19a-3p and Xist. As shown in Figure 6C, miR-19a-3p was pulled down by biotin-labeled WT XIST but failed to be pulled down by mut-XIST. Xist was pulled down by biotin-labeled WT miR-19a-3p, which was failed by mutation miR-19a-3p (Fig. 6C). The RIP assay showed that miR-19a-3p was enriched in Xist, but the enrichment was not affected in mut Xist group. These data showed that miR-19a-5p targets Xist dierctly. We next used the luciferase assay to confirm the target role between lncRNA Xist and miR-19a-3p. As shown in Figure $6 \mathrm{E}, \mathrm{miR}-19 \mathrm{a}-3 \mathrm{p}$ evidently reduced the luciferase activities in Xist group but failed to affect the luciferase activity in mut Xist group. The Xist expression was similar in BMSCs from aged (12 months) WT, miR-19a$3 \mathrm{p}$ tg and KO mice (Supplementary Fig. 1B). Together, these results indicated the direct binding role between lncRNA and miR-19a-3p in BMSCs.

A

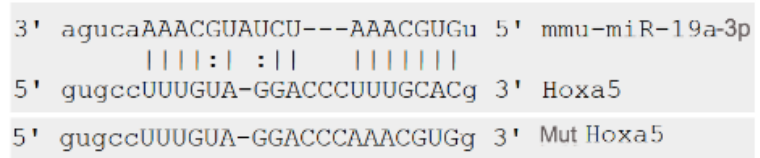

B
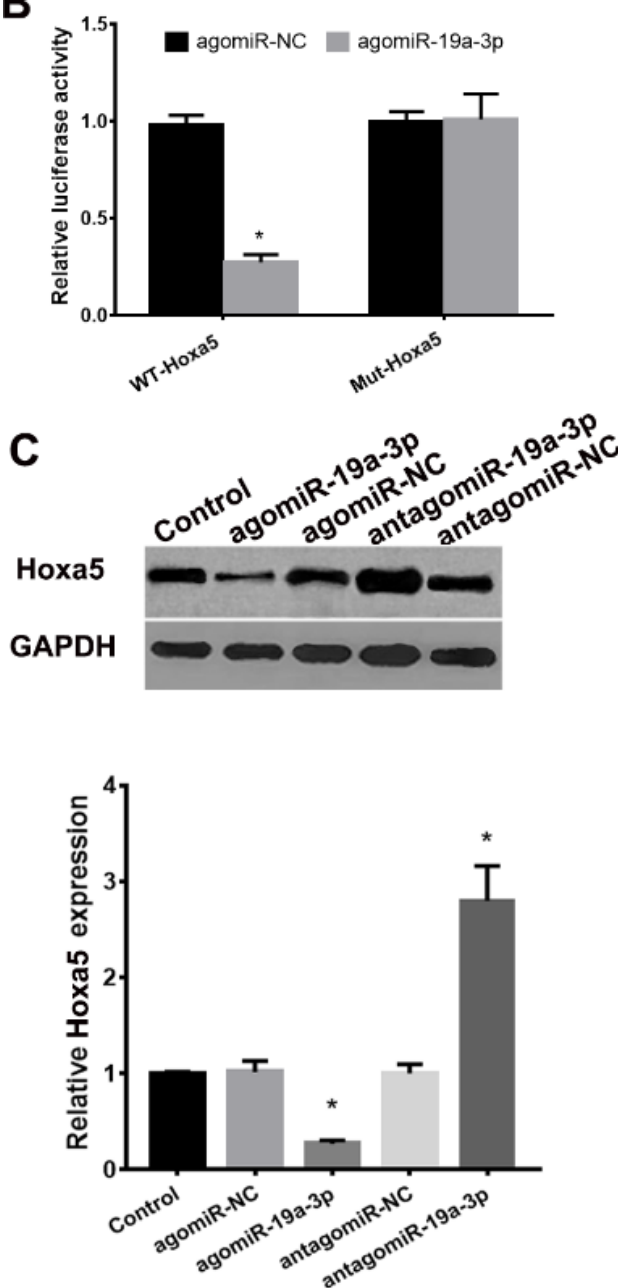

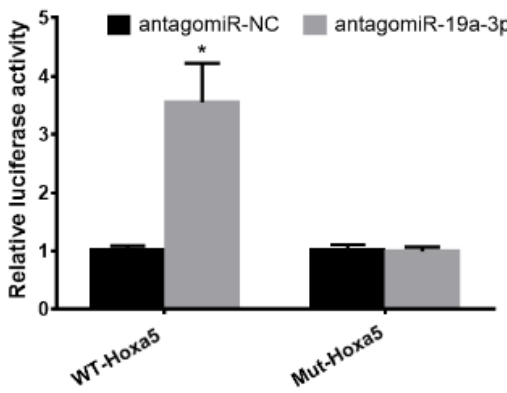

D

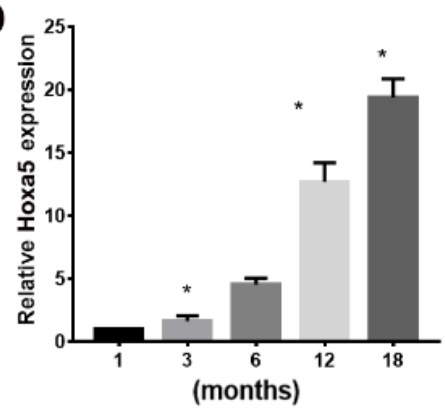

E

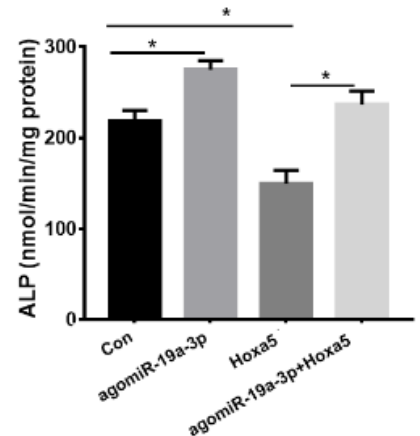

$\mathbf{F}$

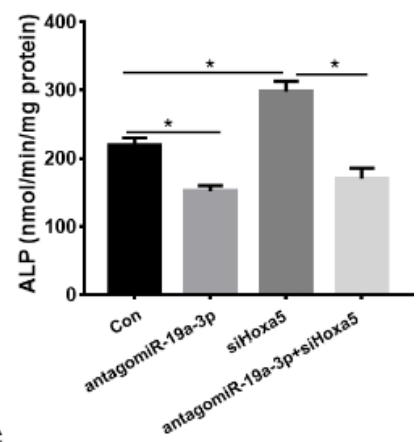

G

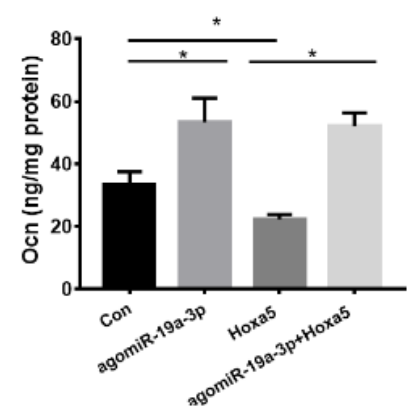

H

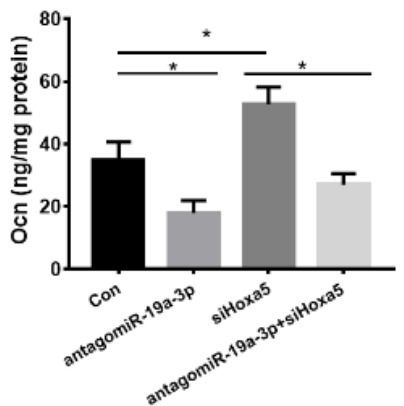

Figure 5. miR-19a-3p regulated Hoxa5 expression by targeting the 3'UTR of Hoxa5 directly. (A) the predict binding role of miR-19a-3p on 3'-UTR of Hoxa5. (B) luciferase reporter assay revealed the target role of miR-19a-3p on Hoxa5. (C) miR-19a-3p regulated Hoxa5 expression. (D) Hoxa5 expression was increased in BMSCs of the aging mice. (E, F) The role of Hoxa5 and miR$19 \mathrm{a}-3 \mathrm{p}$ on ALP activity of BMSCs. (G, F) The role of Hoxa5 and miR-19a-3p on osteocalcin secretion of BMSCs. Data shown as mean \pm SD. $* \mathrm{p}<0.05$. 
A

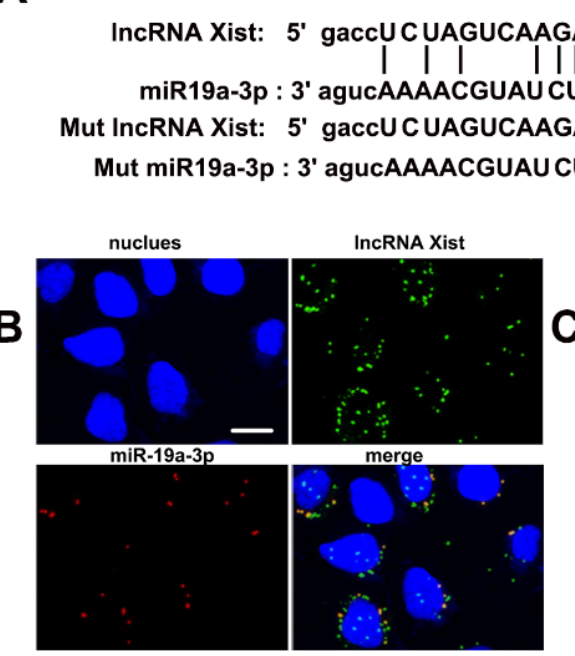

$\mathbf{E}$

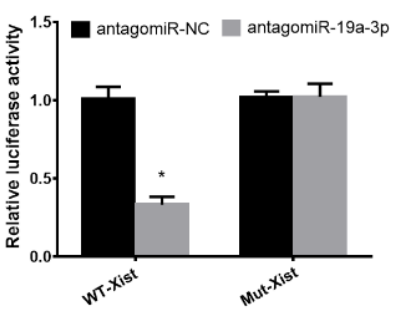

G

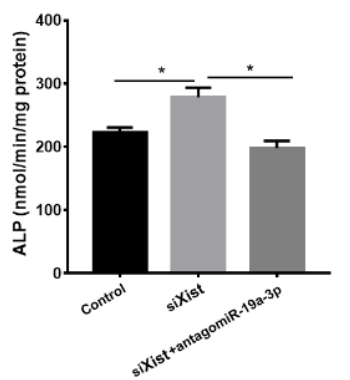

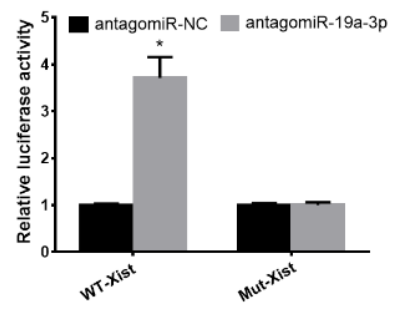

H

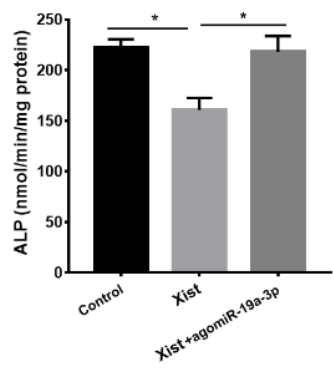

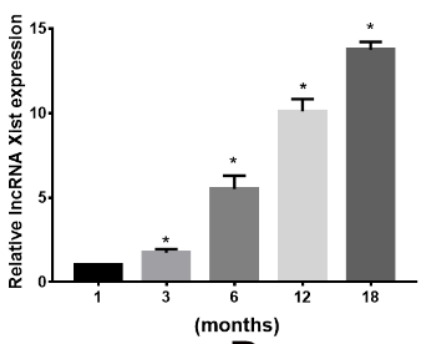

D

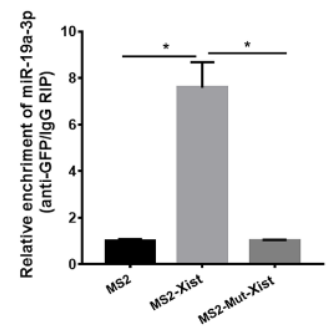

$\mathbf{F}$
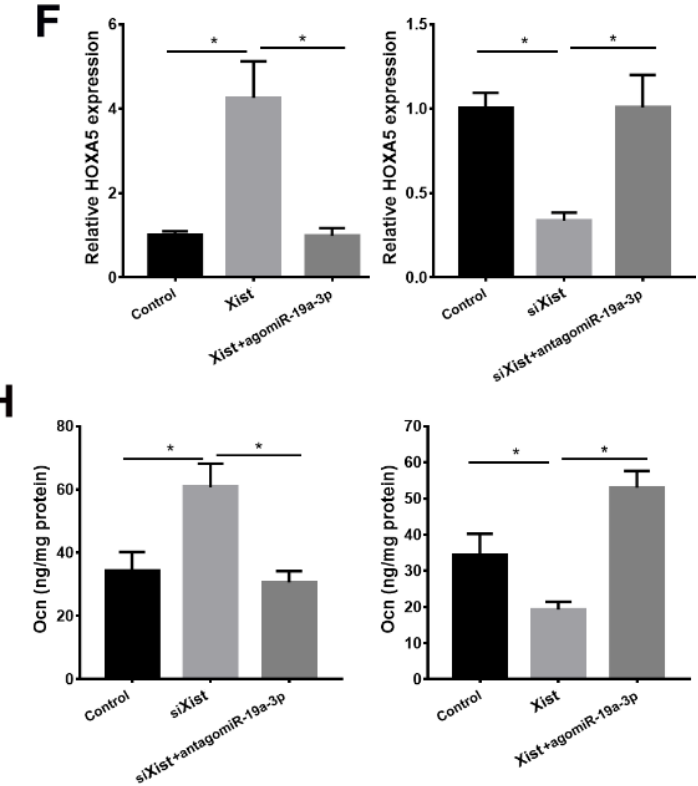

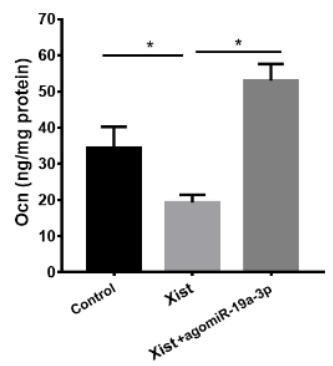

Figure 6. LncRNA Xist was involved osteoblast differentiation via miR-19a-3p/Hoxa5 pathway. (A) Xist expression in BMSCs from mice with different age. (B) RNA FISH assay for miR-19a-3p and Xist. Scale bar=10 $\mu \mathrm{m}$. (C) BMSCs were incubated with biotin-labeled miR-19a-3p and Xist, and qPCR analyses revealed the production of pulled down. (D) RIP assay to reveal the relationship between miR-19a-3p and Xist. (E) Luciferase reporter assay revealed the target role of miR-19a-3p and Xist. (F) The role of Xist and miR-19a-3p on Hoxa5 expression in BMSCs. (G) The effects of Xist and miR-19a-3p on ALP activity. H, The role of Xist and miR-19a-3p on osteocalcin secretion in BMSCs. Data shown as mean \pm SD. $* \mathrm{p}<0.05$.

To determine the regulation of lncRNA Xist on Hoxa5, we transfected pcDNA3.1-XIST to overexpress Xist in miR-19a-3p-overexpressed BMSCs and transfected siRNA Xist to silence Xist in miR-19a-3pknockdown BMSCs. As shown in Figure 6F, overexpressed Xist evidently induced Hoxa5 expression, which was reduced by overexpressed miR-19a-3p. Conversely, silenced Xist reduced Hoxa5 expression, which was abrogated by silenced miR-19a-3p. Taken together, these above results indicated that lncRNA Xist acted as a functional sponge of miR-19a-3p to regulate
Hoxa5 expression in BMSCs. Next, we investigated the function of lncRNA Xist in osteoblast differentiation, by detecting ALP activity and osteocalcin secretion. Enforced Xist reduces ALP activity and osteocalcin secretion of BMSCs, which was abolished by overexpressed miR-19a-3p (Fig. 6G and H). Conversely, silenced Xist induced ALP activity and osteocalcin secretion, which was reversed by antagomiR-19a-3p (Fig. $6 \mathrm{G}$ and $\mathrm{H}$ ). These data collectively indicated that lncRNA Xist was participated in osteoblast differentiation via miR-19a-3p/Hoxa5 pathway. 


\section{DISCUSSION}

BMSCs plays an important role on bone homeostasis via differentiating into osteoblasts or adipocytes. However, the BMSCs tend to differentiate into adipocytes with age, which results in age-related osteoporosis with increased fat and bone loss. Here, we described that miR-19a-3p involved in aging-induced osteoporosis via regulating Hoxa5-mediated BMSCs differentiation.

Recently, several miRNAs have been implicated in the pathogenesis of aged-related osteoporosis [18, 19]. However, the mechanism of miRNAs in aging-induced BMSCs differentiation during osteoporosis remains limited. MiR-19a-3p is a members of the miR-17-92 cluster, and usually reported as oncogene in various cancers, including lung cancer [13, 20], colorectal cancer [21], breast cancer [10], hepatocellular carcinoma[12] and cervical cancer [22]. Studies also showed that miR-19a$3 \mathrm{p}$ involved in the vascular inflammation of atherosclerosis [7] and involved in the epithelium repair of asthma by regulating proliferation of bronchial epithelial cells [23]. Recently, study demonstrated miR$19 b-3 p$ as an important predictive marker of circulating miRNA for aging [24]. miR-17, a member of miR-17-92 cluster, was reported to act as positive regulator of osteogenesis in an inflammatory microenvironment [25]. However, the potential role of miR-19a-3p in aginginduced osteoporosis still remain elusive. In this study, miR-19a-3p expression was remarkably reduced in BMSCs from aged mice and human participants. Furthermore, miR-19a-3 $\mathrm{p}^{-/-}$mice worsen aging-induced osteoporosis with increased bone loss, and miR-19a-3p transgenic mice attenuated aging-induced bone loss. Moreover, miR-19a-3p induced the osteogenic differentiation of BMSCs in vitro. These results demonstrated the important role of miR-19a-3p in aginginduced osteoporosis by regulating BMSCs osteogenic differentiation.

miRNA was reported to involve in various biological processes through regulating the expression of mRNA by targeting their 3' UTR. Previously, miR-19a-3p was involved in tumorigenesis of cervical carcinoma by targeting CUL5 [22]. miR-19a-3p also reported to be involved in the proliferation of colorectal cancer by targeting TIA1 [26] and regulates progression of glioma by targeting RUNX3 directly [27]. Recent study showed that miR-19a-3p regulated macrophage polarization by targeting Fra-1 [6]. Here, we revealed that Hoxa5 was direct target of miR-19a-3p and contribute to miR-19a$3 p$-induced BMSC osteogenic differentiation. Hoxa5 was served as tumor suppressor in a number of cancers including non-small cell lung cancer [28], osteosarcoma [29], gastric cancer [30] and gastric cancer [31]. Hoxa5 was also reported to alleviate inflammation and induce adipose tissue browning [32]. Recently, it was shown that Hoxa5 promoted adipocytes differentiation in primary adipocytes [33]. In this study, we reported that Hoxa5 participated in the miR-19a-3p-mediated switch of BMSCs to osteoblasts.

lncRNAs, a class of $>200$ nucleotides non-coding RNAs, was reported to be involved in various progression of disease including osteoporosis [34-36]. For example, Yang et al. showed that lncRNA ORLNC1 involved in osteoporosis via regulating BMSCs osteogenic and adipogenic differentiation [37]. Liu et al. showed that Lnc-AK077216 promotes osteoclastogenesis via regulating NFATc1 expression [36]. Wang et al. demonstrated lncRNA ODSM as a regulator of osteoblast differentiation and apoptosis via targeting miR-139-3p in osteoblasts [19]. Xist was initially reported as a lncRNA, involving in $\mathrm{X}$ chromosome inactivation [38]. Increasing evidences showed that lncRNA Xist was reported as an oncogene in various tumors $[39,40]$. Moreover, recent studies indicated the involvement of lncRNA Xist in myocardial infarction [41]. In this study, IncRNA Xist serve as a sponge of miR-19a-3p in BMSCs, and lncRNA Xist regulated Hoxa5 expression and BMSCs osteogenic differentiation via targeting miR-19a-3p directly.

In conclusion, our finding indicated that miR-19a-3p expression was downregulated in BMSCs with aged and the exogenous miR-19a-3p in BMSCs promoted osteogenic differentiation and bone formation partly by targeting Hoxa5 directly. Moreover, IncRNA Xist function as the sponge of miR-19a-3p and was involved in osteogenic differentiation. These results demonstrated the function of the lncRNA Xist/miR-19a-3p /Hoxa5 signaling pathway in aging-induced BMSCs osteogenic differentiation, revealing a potential therapeutic target for osteoporosis.

\section{Acknowledgments}

This study was supported by National Natural Science Foundation of China (Grant Nos. 81772866); National Natural Science Foundation of China (Grant Nos. 81502331); Natural Science Foundation of Hunan Province (Grant Nos. 2018JJ2617); Natural Science Foundation of Hunan Province (Grant Nos. 2016JJ3176).

\section{Conflicts of Interest}

The authors declare no conflict of interest.

\section{Supplementary Materials}

The Supplemenantry data can be found online at: www.aginganddisease.org/EN/10.14336/AD.2019.0724. 


\section{References}

[1] Collison J (2017). Bone: miR-106b promotes osteoporosis in mice. Nat Rev Rheumatol, 13:130.

[2] Jing H, Su X, Gao B, Shuai Y, Chen J, Deng Z, et al. (2018). Epigenetic inhibition of Wnt pathway suppresses osteogenic differentiation of BMSCs during osteoporosis. Cell Death Dis, 9:176.

[3] Li CJ, Cheng P, Liang MK, Chen YS, Lu Q, Wang JY, et al. (2015). MicroRNA-188 regulates age-related switch between osteoblast and adipocyte differentiation. J Clin Invest, 125:1509-1522.

[4] Guo Q, Chen Y, Guo L, Jiang T, Lin Z (2016). miR-23a/b regulates the balance between osteoblast and adipocyte differentiation in bone marrow mesenchymal stem cells. Bone Res, 4:16022.

[5] Huang J, Zhao L, Xing L, Chen D (2010). MicroRNA204 regulates Runx2 protein expression and mesenchymal progenitor cell differentiation. Stem Cells, 28:357-364.

[6] Yang J, Zhang Z, Chen C, Liu Y, Si Q, Chuang TH, et al. (2014). MicroRNA-19a-3p inhibits breast cancer progression and metastasis by inducing macrophage polarization through downregulated expression of Fra-1 proto-oncogene. Oncogene, 33:3014-3023.

[7] Chen H, Li X, Liu S, Gu L, Zhou X (2017). MircroRNA$19 \mathrm{a}$ promotes vascular inflammation and foam cell formation by targeting HBP-1 in atherogenesis. Sci Rep, $7: 12089$.

[8] Wang N, Li R, Xue M (2019). Potential regulatory network in the PSG10P/miR-19a-3p/IL1RAP pathway is possibly involved in preeclampsia pathogenesis. J Cell Mol Med, 23:852-864.

[9] Qiao F, Gong P, Song Y, Shen X, Su X, Li Y, et al. (2018). Downregulated PITX1 Modulated by MiR-19a-3p Promotes Cell Malignancy and Predicts a Poor Prognosis of Gastric Cancer by Affecting Transcriptionally Activated PDCD5. Cell Physiol Biochem, 46:2215-2231.

[10] Lee S, Lee H, Bae H, Choi EH, Kim SJ (2016). Epigenetic silencing of miR-19a-3p by cold atmospheric plasma contributes to proliferation inhibition of the MCF-7 breast cancer cell. Sci Rep, 6:30005.

[11] Zhang B, Liu Y, Zhang J (2019). Silencing of miR-19a$3 p$ enhances osteosarcoma cells chemosensitivity by elevating the expression of tumor suppressor PTEN. Oncol Lett, 17:414-421.

[12] Wang Z, Shi Z, Zhang L, Zhang H, Zhang Y (2019). Profilin 1 , negatively regulated by microRNA-19a-3p, serves as a tumor suppressor in human hepatocellular carcinoma. Pathol Res Pract, 215:499-505.

[13] Gu Y, Liu S, Zhang X, Chen G, Liang H, Yu M, et al. (2017). Oncogenic miR-19a and miR-19b co-regulate tumor suppressor MTUS1 to promote cell proliferation and migration in lung cancer. Protein Cell, 8:455-466.

[14] Yang M, Li CJ, Sun X, Guo Q, Xiao Y, Su T, et al. (2017). MiR-497 approximately 195 cluster regulates angiogenesis during coupling with osteogenesis by maintaining endothelial Notch and HIF-1alpha activity. Nat Commun, 8:16003.
[15] Iliopoulos D, Bimpaki EI, Nesterova M, Stratakis CA (2009). MicroRNA signature of primary pigmented nodular adrenocortical disease: clinical correlations and regulation of Wnt signaling. Cancer Res, 69:3278-3282.

[16] Wang R, Zhang S, Chen X, Li N, Li J, Jia R, et al. (2018). CircNT5E Acts as a Sponge of miR-422a to Promote Glioblastoma Tumorigenesis. Cancer Res, 78:4812-4825.

[17] Cheng X, Zhang L, Zhang K, Zhang G, Hu Y, Sun X, et al. (2018). Circular RNA VMA21 protects against intervertebral disc degeneration through targeting miR200c and X linked inhibitor-of-apoptosis protein. Ann Rheum Dis, 77:770-779.

[18] Liao L, Su X, Yang X, Hu C, Li B, Lv Y, et al. (2016). TNF-alpha Inhibits FoxO1 by Upregulating miR-705 to Aggravate Oxidative Damage in Bone Marrow-Derived Mesenchymal Stem Cells during Osteoporosis. Stem Cells, 34:1054-1067.

[19] Wang Y, Wang K, Hu Z, Zhou H, Zhang L, Wang H, et al. (2018). MicroRNA-139-3p regulates osteoblast differentiation and apoptosis by targeting ELK1 and interacting with long noncoding RNA ODSM. Cell Death Dis, 9:1107.

[20] Zhu J, Wang S, Chen Y, Li X, Jiang Y, Yang X, et al. (2017). miR-19 targeting of GSK3beta mediates sulforaphane suppression of lung cancer stem cells. J Nutr Biochem, 44:80-91.

[21] Wang T, Xu X, Xu Q, Ren J, Shen S, Fan C, et al. (2017). miR-19a promotes colitis-associated colorectal cancer by regulating tumor necrosis factor alpha-induced protein 3-NF-kappaB feedback loops. Oncogene, 36:3240-3251.

[22] Xu XM, Wang XB, Chen MM, Liu T, Li YX, Jia WH, et al. (2012). MicroRNA-19a and -19b regulate cervical carcinoma cell proliferation and invasion by targeting CUL5. Cancer Lett, 322:148-158.

[23] Haj-Salem I, Fakhfakh R, Berube JC, Jacques E, Plante S, Simard MJ, et al. (2015). MicroRNA-19a enhances proliferation of bronchial epithelial cells by targeting TGFbetaR2 gene in severe asthma. Allergy, 70:212-219.

[24] Margolis LM, Lessard SJ, Ezzyat Y, Fielding RA, Rivas DA (2017). Circulating MicroRNA Are Predictive of Aging and Acute Adaptive Response to Resistance Exercise in Men. J Gerontol A Biol Sci Med Sci, 72:1319-1326.

[25] Liu Y, Liu W, Hu C, Xue Z, Wang G, Ding B, et al. (2011). MiR-17 modulates osteogenic differentiation through a coherent feed-forward loop in mesenchymal stem cells isolated from periodontal ligaments of patients with periodontitis. Stem Cells, 29:1804-1816.

[26] Liu Y, Liu R, Yang F, Cheng R, Chen X, Cui S, et al. (2017). miR-19a promotes colorectal cancer proliferation and migration by targeting TIA1. Mol Cancer, 16:53.

[27] Sun J, Jia Z, Li B, Zhang A, Wang G, Pu P, et al. (2017). MiR-19 regulates the proliferation and invasion of glioma by RUNX3 via beta-catenin/Tcf-4 signaling. Oncotarget, 8:110785-110796.

[28] Chang CJ, Chen YL, Hsieh CH, Liu YJ, Yu SL, Chen JJW, et al. (2017). HOXA5 and p53 cooperate to suppress lung cancer cell invasion and serve as good 
prognostic factors in non-small cell lung cancer. J Cancer, 8:1071-1081.

[29] Chen YQ, Yang TQ, Zhou B, Yang MX, Feng HJ, Wang YL (2019). HOXA5 overexpression promotes osteosarcoma cell apoptosis through the p53 and p38alpha MAPK pathway. Gene, 689:18-23.

[30] Peng X, Zha L, Chen A, Wang Z (2018). HOXA5 is a tumor suppressor gene that is decreased in gastric cancer. Oncol Rep, 40:1317-1329.

[31] Wu Y, Zhou T, Tang Q, Xiao J (2019). HOXA5 inhibits tumor growth of gastric cancer under the regulation of microRNA-196a. Gene, 681:62-68.

[32] Cao W, Huang H, Xia T, Liu C, Muhammad S, Sun C (2018). Homeobox a5 Promotes White Adipose Tissue Browning Through Inhibition of the Tenascin C/TollLike Receptor 4/Nuclear Factor Kappa B Inflammatory Signaling in Mice. Front Immunol, 9:647.

[33] Cao W, Xu Y, Luo D, Saeed M, Sun C (2018). Hoxa5 Promotes Adipose Differentiation via Increasing DNA Methylation Level and Inhibiting PKA/HSL Signal Pathway in Mice. Cell Physiol Biochem, 45:1023-1033.

[34] Mei B, Wang Y, Ye W, Huang H, Zhou Q, Chen Y, et al. (2019). LncRNA ZBTB40-IT1 modulated by osteoporosis GWAS risk SNPs suppresses osteogenesis. Hum Genet, 138:151-166.

[35] Hu K, Jiang W, Sun H, Li Z, Rong G, Yin Z (2019). Long noncoding RNA ZBED3-AS1 induces the differentiation of mesenchymal stem cells and enhances bone regeneration by repressing IL-1beta via Wnt/betacatenin signaling pathway. J Cell Physiol.

[36] Liu C, Cao Z, Bai Y, Dou C, Gong X, Liang M, et al. (2019). LncRNA AK077216 promotes RANKL-induced osteoclastogenesis and bone resorption via NFATc1 by inhibition of NIP45. J Cell Physiol, 234:1606-1617.

[37] Yang L, Li Y, Gong R, Gao M, Feng C, Liu T, et al. (2019). The Long Non-coding RNA-ORLNC1 Regulates Bone Mass by Directing Mesenchymal Stem Cell Fate. Mol Ther, 27:394-410.

[38] Penny GD, Kay GF, Sheardown SA, Rastan S, Brockdorff N (1996). Requirement for Xist in $X$ chromosome inactivation. Nature, 379:131-137.

[39] Li C, Wan L, Liu Z, Xu G, Wang S, Su Z, et al. (2018). Long non-coding RNA XIST promotes TGF-betainduced epithelial-mesenchymal transition by regulating miR-367/141-ZEB2 axis in non-small-cell lung cancer. Cancer Lett, 418:185-195.

[40] Chen YK, Yen Y (2019). The Ambivalent Role of lncRNA Xist in Carcinogenesis. Stem Cell Rev, 15:314323.

[41] Zhou T, Qin G, Yang L, Xiang D, Li S (2019). LncRNA XIST regulates myocardial infarction by targeting miR130a-3p. J Cell Physiol, 234:8659-8667. 